SHS Web of Conferences 20, 01004 (2015)

DOI: $10.1051 /$ shsconf $/ 20152001004$

(C) Owned by the authors, published by EDP Sciences, 2015

\title{
L'exercice de (re)présentation de soi lors d'une enquête de terrain : négociation ? Enjeu épistémologique ?
}

\section{(Re)presenting the self during a field survey: negotiation? Epistemological stake?}

Myriam Dupouy ${ }^{1, a}$

${ }^{1}$ CRBC EA 4451, 20, rue Duquesne - CS 93837, 29238 Brest Cedex 3, France

\begin{abstract}
Résumé. Dans le cadre de notre recherche doctorale portant sur la prise en compte des représentations et attitudes liées à la musicalité de la langue française en formation FLI pour adultes en situation de migration, nous avons mené une enquête de terrain de type ethnosociolinguistique (Blanchet, 2000 [1]). Comme dans toute entrée en matière, la question de la posture à adopter s'est posée rapidement, tout d'abord en termes de pratique : comment prendre contact avec les organismes ? Ou plutôt, par quelle « entrée » se présenter ? Comment conservet-on son intégrité lorsqu'on essaye de s'adapter? Est-il scientifiquement possible de se saisir de ses compétences plurielles pour sa recherche ? Humainement, peut-on être des deux côtés de la barrière symbolique souvent existante entre enseignants et apprenants? Il semble donc nécessaire de s'adapter, négocier sans cesse avec les personnes du terrain, inventer les conduites à adopter avec les autres, mais aussi avec soi-même La négociation de la (re)présentation du jeune chercheur se présente alors sous la forme d'une co-construction, entre négociations et réflexion épistémologique.
\end{abstract}

\begin{abstract}
Our doctoral research tackles with the understanding of the representations and the attitudes in relation to the musicality of the French language, in French as a social integration language (FLI) training courses for adult migrants. In that context, we have driven a field survey in an ethno-sociolinguistical approach (Blanchet, 2000 [1]). As in any first steps on the field, the issue of self-presentation has quickly arisen. First, in practical terms: how to initiate a contact with organisations? Or rather, by which "entry" could we introduce ourselves? How to maintain our integrity while trying to adapt? Would it be scientifically relevant to use our plural skills on the field? Personally, could we position ourselves on both sides of the symbolic gap, which often exists between teachers and learners? It thus seems necessary to adapt, to negotiate relentlessly with individuals on the field, to invent behaviours to adopt with others and with ourselves as well. Then, negotiating the (re)presentation of the junior researcher is performed by coconstruction, between negotiations and epistemological questioning.
\end{abstract}

\section{Introduction}

Cette contribution vise à questionner les modalités d'approche du terrain de recherche, avec l'intention de mettre en forme des réflexions cycliques et transversales liées au travail de thèse. Le travail de terrain est très régulièrement cité dans les travaux scientifiques afin de présenter et d'étayer l'enquête, mais son approche reste souvent discrète, alors que les questions liées à l'entrée en matière du jeune chercheur sur le terrain (Goffman, 1973 [2], Becker, 2002 [3], Paillé \& Mucchielli, 2012 [4]), sur les différents modes de négociation, d'insertion dans le terrain prédéfini cristallisent régulièrement les échanges lors des rencontres entre jeunes chercheurs.

\footnotetext{
a Auteur de correspondance : myriam.dupouy@univ-brest.fr
}

This is an Open Access article distributed under the terms of the Creative Commons Attribution License 4.0, which permits unrestricted use, distribution, and reproduction in any medium, provided the original work is properly cited. 
Ma recherche doctorale, située au carrefour de la sociolinguistique et de la didactique des langues, porte sur les représentations et attitudes liées à la musicalité de la langue française en formation FLI ${ }^{\mathrm{b}}$, Français Langue d'Insertion/d'Intégration, pour adultes en situation de migration en Bretagne.

Qu'est-ce que j'entends par musicalité de la langue ? Musicalité signifie ici accent, dans son sens commun et en tant que concept sociolinguistique. Ainsi, ce dernier implique que les discours et les représentations qui lui sont attachés le caractérisent comme marqueur social (Meyer, 2012 [5]) et identitaire. Ainsi, pour reprendre les propos de Médéric Gasquet Cyrus, « Non seulement les accents ne sont pas réductibles, pour les locuteurs, à des prononciations (car leur conception de l'accent déborde ce niveau de description), mais en outre l'accent est inscrit dans une relation d'altérité et de pouvoir, prenant ainsi une dimension éthique et politiquement incontournable » (Gasquet-Cyrus, 2010 [6]).

J'analyse des usages et des représentations, du point de vue de la perception et de l'enseignement afin de comprendre les interactions, les jeux de pouvoir symboliques, les jugements de valeur, les sentiments d'insécurité/sécurité linguistique qui existent au sein de ces formations Français langue d'Insertion/d'intégration.

Les questionnements qui animent ma recherche s'orientent vers les problématiques du plurilinguisme, de l'acceptation (ou non) de l'hétérogénéité des accents, de la hiérarchisation des langues, et d'un point de vue plus didactique, des moyens dont disposent les acteurs du champ pour travailler ce que j'appelle la musicalité et ses représentations.

A ce stade de la recherche, j'ai réalisé une enquête de terrain ${ }^{c}$ consistant en l'observation des formations FLI en contexte-classe, passation de questionnaires exploratoires et entretiens avec les stagiaires et les formatrices. L'analyse de cette enquête nous permet d'identifier un sentiment d'illégitimité en tant que locuteur pour certains stagiaires. Il semble en effet que ces derniers perçoivent l'usage/apprentissage de la langue française paradoxalement comme nécessaire pour s'insérer (alors reflet d'un discours dominant), mais aussi potentiellement vecteur d'un « abandon » de ce qu'ils placent comme étant une part de leur identité, dans ce cas leurs langues premières. En ce qui concerne leurs représentations des accents, le français serait d'ordre monophonique, «pas fort », «poli » puisque non accentué selon eux, alors que leurs langues premières seraient accentuées, «vivantes », " fortes » et donc, moins normées.

Par ailleurs, certains stagiaires expliquent que leurs accents, leurs langues « contamineraient » (pour reprendre le terme d'une stagiaire) leurs enfants et les empêcheraient de mener une vie «normale » en France, et plus particulièrement en contexte scolaire. A nouveau, les sentiments d'insécurité linguistique, d'illégitimité liés à une hiérarchisation des langues au sein du répertoire plurilingue sont les premières pistes d'analyse qui se dégagent du travail de terrain.

Cette recherche s'appuie sur un travail de terrain que je contextualiserai puis analyserai sous l'angle de l'exercice de (re)présentation de soi en tant que jeune chercheur dans l'environnement particulier de la formation linguistique obligatoire pour adultes migrants.

\section{Le contexte de l'enquête}

J'ai mené une enquête de terrain en région Bretagne où nous comptons cinq organismes labellisés qui dispensent des formations linguistiques FLI. Ces formations présentent un caractère obligatoire dans le cadre du Contrat d'Accueil et d'intégration ${ }^{\mathrm{d}}$ qui lie les adultes migrants présentant un niveau en langue française insuffisant aux yeux de l'OFII, l'Office français de l'immigration et de l'intégration ${ }^{\mathrm{e}}$.

\footnotetext{
b Pour la démarche Français langue d'intégration ainsi que le Label qualité FLI, voir http://www.immigration.interieur.gouv.fr/Accueil-et-accompagnement/L-apprentissage-du-francais/Le-labelqualite-Francais-langue-d-integration/Le-label-qualite-Francais-langue-d-integration.

${ }^{c}$ Pour de plus de précisions sur les outils méthodologiques, voir infra p.3.

${ }^{d}$ Le Contrat d'Accueil et d'Intégration est obligatoire depuis 2007, il comprend une formation civique, une formation linguistique (selon critères établis par le Ministère), une session d'information sur la vie en France, un bilan de compétences et un accompagnement social (idem).

e L'Office français de l'immigration et de l'intégration a été crée en 2009, c'est l'opérateur principal de la Direction générale des étrangers en France. URL : http://www.ofii.fr.
} 
L'adulte se voit attribuer un nombre d'heures de formation linguistique qu'il suivra dans une structure (de type institut de formation) labellisée par l'Etat ${ }^{\mathrm{f}}$.

Les lieux d'enquête sont des centres de formation pour adultes. La formation FLI-FLE n'est qu'un pan de leur offre qui est très variée (insertion professionnelle, métiers de la petite enfance, d'aide à la personne). Ces formations sont dispensées par des formatrices ( $\mathrm{j}$ 'indique volontairement ce terme au féminin car je n'ai rencontré que des femmes jusqu'à présent) formées en FLE et en FLI par le biais de formations internes.

Les groupes de stagiaires se composent en moyenne d'une petite quinzaine d'adultes de tous âges et de toutes nationalités. Le terme d'hétérogénéité est certainement celui qui caractérise le mieux le public. En effet, bien que certaines zones d'origine soient plus représentées que d'autres (pays du Caucase et Asie du Sud), j'ai rencontré des stagiaires de plus d'une vingtaine de nationalités. Les femmes sont légèrement plus nombreuses que les hommes, les âges sont très variés, avec des jeunes adultes de 18 ans jusqu'à des stagiaires de plus de 65 ans. Enfin, les profils sociolinguistiques des stagiaires sont très divers, ainsi que les niveaux d'alphabétisation, de scolarisation et de compétences professionnelles en fonction des variables sociales, géographiques et culturelles.

Mon travail de recherche de type ethno-sociolinguistique (Blanchet, 2000 [1]) s'inscrit dans une approche qualitative, et plus particulièrement dans une démarche empirico-inductive. Une réflexion épistémologique coordonnée à une phase exploratoire élargie m'a orientée vers un choix d'outils de recueil d'informations qui sont l'observation participante (Goffman, 1973 [2], Becker, 2002 [3]), le questionnaire, les entretiens semi-directifs (Paillé \& Mucchielli, 2012 [4]) et la biographie langagière (Bretegnier, 2009 [10]), afin d'explorer mes pistes de travail qui découlent des questions ci-dessous.

S'il existe des représentations de la musicalité de la langue française chez les stagiaires, les formateurs, quelles sont-elles ? Comment s'organisent-elles?

En quoi un travail sur les représentations (chez les stagiaires et formateurs) liées à la musicalité de la langue française, langue cible, a-t-il une incidence sur le déroulement de la formation (Moore, 2001 [7]) ?

Quel est le lien entre la volonté d'intégration indiquée par le Ministère de l'Immigration initiateur du projet et les enjeux d'une telle recherche sur l'oral, alors même que «les attentes sociales en maîtrise de la langue apparaissent génératrices d'insécurité linguistique » (Adami, Etienne, Bretegnier, 2010 [8])?

Quelle est la formation des formateurs en FLI aux questions de l'oralité en didactique des langues?

Les concepts d'asymétrie et d'insécurité linguistique peuvent-ils être traités sous l'angle spécifique de l'oralité ? Si oui, de quelle(s) façon(s) un travail sur les représentations contribuerait à des formations linguistiques plus « efficaces »?

\section{Les différentes postures}

Comme dans toute entrée en matière, la question de la posture à adopter s'est posée rapidement, tout d'abord en termes de pratique : comment prendre contact avec les organismes ? Ou plutôt, par quelle «entrée»se présenter? Outre l'aspect proprement logistique des choses, ce premier temps de (re)présentation peut s'avérer décisif pour l'accès au terrain. Pour reprendre l'analyse de Goffman (Goffman, 1973 [2]), cela correspond à une étape préliminaire de «l'entrée en scène », une introduction aux interactions de terrain.

\subsection{L'équipe pédagogique}

Pour les premiers contacts avec les directeurs de ces structures, la présentation d'un projet de recherche universitaire encadré par un professeur au sein d'un laboratoire de recherche, et donc une approche sous

\footnotetext{
${ }^{\mathrm{f}}$ Voir à ce propos : http://www.immigration.interieur.gouv.fr/Accueil-et-accompagnement/L-apprentissage-dufrancais/Le-label-qualite-Francais-langue-d-integration/Liste-des-organismes-de-formation-labellises-Francaislangue-d-integration.
} 
le titre de doctorante, semblait la plus appropriée. Mais si une posture paraît plus adéquate face à un des acteurs du champ étudié, elle ne l'est pas forcément auprès d'autres qui peuvent se voir imposer une présence par leurs supérieurs hiérarchiques.

En effet, lorsque la communication en interne dans la structure est bonne, le directeur informe le responsable pédagogique de ma venue qui informe à son tour les formateurs. Ainsi, j'ai reçu en copie un courriel destiné à plusieurs personnes dont le but était d'informer de ma venue et de décider ensemble des modalités du temps de terrain. Dans un autre contexte, un directeur a également programmé seul les dates de mon accueil, sans que je puisse entrer en contact avec l'équipe pédagogique. Cela a créé un sentiment de surprise lors de mon arrivée, la formatrice n'étant pas informée des dates et raisons de ma venue.

Ce sentiment de surprise peut se doubler de doutes et d'incompréhensions face à ma demande. La recherche semble éloignée, selon certains d'entre eux, de leur réalité. Je l'ai senti à plusieurs reprises, mais on me l'a aussi dit frontalement, ce qui peut déstabiliser (observer au nom de qui ? pour faire quoi ?), tout en ouvrant un espace de discussions intenses.

C'est à ce moment là que j'ai compris que mon expérience professionnelle d'enseignante de FLE à l'étranger et en France était mieux perçue car plus facilement identifiable. Ainsi, d'apprentie chercheure, je suis passée à « enseignante de FLE ».

Cette relation de pairs a instauré une certaine connivence (tutoiement, temps informels, confidences) et si cette "casquette professionnelle » m'a permis d'entrevoir, d'entendre et surtout d'échanger en profondeur avec les formatrices, elle m'a également posé question : selon elles, je peux les remplacer pour un temps, aider, faire des transmissions aux collègues, bref, être formatrice FLI. Et donc me placer d'un côté de la barrière symbolique enseignant/apprenant.

De plus, le formateur peut craindre un jugement de son activité de la part d'une collègue, et orienter le contenu de son cours. A nouveau, un changement, ou un réajustement de posture s'impose afin de rappeler l'anonymat d'une recherche scientifique, l'absence de jugements de valeur, la volonté de saisir leur réalité en essayant d'atténuer la lourdeur d'une présence extérieure.

Sur un des sites, j'ai été introduite par la responsable comme étudiante de FLE (sans que je sois au courant), donc certainement en stage préprofessionnel pour devenir enseignante : lors de mon premier jour, on m'a expliqué, détaillé, le contenu pédagogique, et on a fini par me demander d'envoyer une copie de mon rapport de stage pour archives.

C'est seulement à ce moment que j'ai compris que la communication n'avait pas été optimale, loin de là, et lorsque j'ai enfin pu prendre le temps de me présenter comme enseignante FLE en doctorat, donc en reprise d'études, les comportements ont énormément changé. En effet, ce terme de reprise d'études a trouvé un écho auprès de certaines formatrices qui ont opéré un rapprochement par rapport à leur propre cursus de formation. Certaines d'entre elles étaient obligées de suivre une formation FLI, ou sur les savoirs de base afin de répondre au cahier des charges imposé par le Label FLI. Une responsable pédagogique qui était en reprise d'études en master rapprochait nos situations par la phrase récurrente « on est dans la même galère », ce qui a instauré une certaine connivence, et même solidarité lors de nos rencontres. On m'a alors ouvert les portes des lieux réservés aux formateurs, proposé de déjeuner entre formatrices, et même informé d'éventuels dysfonctionnements de la structure.

\subsection{Les stagiaires}

Prendre place en salle de formation aux côtés des stagiaires pendant plusieurs semaines est une des manières de faire de l'observation participante. Mais si cet outil de recueil de données instaure une certaine familiarité avec les formateurs et les stagiaires, le statut de pair peut difficilement être instauré avec les adultes en formation linguistique. Il faut alors à nouveau réussir à "faire son entrée » et redéfinir sa posture.

Ainsi, la posture de l'étudiante qui étudie un groupe-classe pour ses études est un moyen de se placer de « leur côté », c'est-à-dire en situation d'apprentissage (en situation de pair, d'un côté de la barrière symbolique). J'ai souvent été interrogée sur ma vie personnelle, mes études, et j'ai été invitée à partager des temps hors-classe. Ces temps en dehors de la salle, comme par exemple des sorties organisées à 
l'occasion d'un forum pour l'emploi ou du Printemps du cinéma sont l'occasion de monter à plusieurs en voiture, de faire un bout de chemin ensemble.

Souvent, le rire est très présent, et il me semble être un moyen de négocier son rapport à l'Autre, tout en étant un élément illustrant le lien entre l'empirique et l'épistémologie, car comme toute donnée, il peut faire l'objet d'une analyse et venir étayer notre appareil conceptuel.

La sociologue Annick Madec écrit que : «Le rire avec est le rire partagé dans les espaces privés, là où les rapports entre les individus affectent d'être égalitaires. L'humour sert bien souvent à montrer que personne n'est dupe, que l'égalité n'est que supposée. Il sert à montrer l'ambivalence, les différences, la complexité. Il fragmente le " nous » d'appartenance » (Madec, 2002 [9]).

Je pense par exemple à trois mères qui n'ont cessé de vouloir me présenter leurs fils afin d'organiser des mariages en France et dans leurs pays. Ces discussions ont provoqué beaucoup de rires, et ont été propices à la discussion : pourquoi vouloir marier leurs fils à une Française ? Un désir de rester vivre en France pour un long moment? Alors que nous riions, le ton est monté entre deux mères, l'une disant qu'il fallait une femme de la même nationalité et de la même religion, l'autre lui répondant que se marier avec une française était bénéfique en tous points : les papiers, mais aussi la langue. Ici, le facteur rire m'a permis d'ajouter une dimension genrée à ma posture. Bien que la réflexion sur le genre n'entre pas directement dans le cadre de ma recherche, le fait de rencontrer uniquement des formatrices, de rencontrer davantage de stagiaires femmes qu'hommes, d'être moi-même une femme sont autant de données qui pourront venir nourrir les analyses ${ }^{\mathrm{g}}$.

Mais alors qu'une posture semble s'instaurer au fil du temps, il y a à nouveau un basculement : les stagiaires avec qui j'ai le plus échangé, ri, me sollicitent régulièrement pour remplir leurs papiers administratifs, appeler différentes structures, comprendre certains courriers... Ces sollicitations étaient plus nombreuses dans les structures où les formatrices avaient un fonctionnement qui séparait la formation pédagogique de la vie courante. Cette nouvelle «casquette » de native, je ne l'avais pas anticipée, ce qui fait que j'ai été quelque peu «débordée » par ces demandes nombreuses. Mais en prenant du recul, j'en ai déduit de nouveaux éléments, me permettant de mieux cerner et comprendre les difficultés des stagiaires à gérer une administration lourde et des formatrices fatiguées de devoir remplir sur leur temps de pause des formulaires en tout genre.

Ces relations d'échanges symboliques permettent de gagner la confiance nécessaire au bon déroulement des entretiens de type compréhensif, notamment lorsqu'il s'agit d'approches de type « biographie langagière » et « histoire de langues ». Cette dernière approche est ainsi définie par Aude Bretegnier : "l'histoire de langues renvoie à l'histoire, en cours et en devenir, des relations complexes, ambivalentes, que les individus sociaux construisent et entretiennent avec leur(s) langue(s). Elle comprend l'histoire des images, des statuts, des légitimités que les locuteurs associent aux langues, dont ils les dotent; l'histoire de leurs sentiments vis-à-vis de ces langues et vis-à-vis d'eux-mêmes comme locuteurs, plus ou moins légitimés à se les approprier, à y prendre la parole, à s'y produire » (Bretegnier, 2009 [10]).

Il s'agit d'un outil de co-construction entre les deux interlocuteurs en présence, pertinent pour comprendre, saisir les représentations, les phénomènes de transmissions et les modes d'apprentissage des différentes langues qui composent ce qu'on appelle un répertoire plurilingue. Même si chaque histoire de langue se construit d'un point de vue méthodologique avec des spécificités liées au thème de recherche, on retrouve des éléments phares tels que le « (...) parcours de locuteur, d'apprenant, de transmetteur de langues, de l'histoire-contextes de la construction de ses relations aux langues » (Bretegnier, 2009 [10]).

La finalité de l'utilisation de cet outil méthodologique est ici de mener conjointement un travail de réflexivité afin de permettre la « construction par (et avec) un individu social, d'un récit contextualisant de l'histoire relationnelle et socio-interactionnelle à ses langues, et dans ses langues » (Bretegnier, 2012 [11]).

\footnotetext{
$\mathrm{g}$ Voir à ce propos les travaux de Nadia Ouabdelmoumen, notamment sa thèse, Contractualisation des rapports sociaux : le volet linguistique du contrat d'accueil et d'intégration au prisme du genre sous la direction de Philippe Blanchet soutenue à Rennes 2 le 09-12-2014.
} 
Cette démarche nécessite un climat de confiance, qui est lié au facteur temporel. En effet, comment entamer un échange avec quelqu'un qu'on vient à peine de rencontrer? Cette notion temporelle, inhérente au travail de terrain, est à mon avis en relation avec le statut même du jeune chercheur, dans le sens où il est institutionnellement tenu par un calendrier souvent très court lors de ses recherches sur le terrain.

Alors, quelles sont les limites de ces différentes postures, et en quoi le fait d'être cataloguée comme formatrice, stagiaire, étudiante joue un rôle dans le travail de terrain?

Ce que j'ai remarqué lors de la phase exploratoire, c'est que les premiers contacts étaient difficilement réajustables : la manière d'être identifiée par les acteurs du champ semble figée. Alors que l'objectif est d'entrer en contact et d'instaurer un climat de confiance pour la discussion, une posture mal gérée peut venir ternir cet objectif. Cela m'est arrivé lorsque l'on m'a par exemple demandé de faire les transmissions avec la formatrice remplaçante. Une fois ce temps de transmission et d'explications pratiques sur le fonctionnement du groupe passé, je n'ai plus eu le même rapport avec les stagiaires. Ceci signifie que cette posture a bloqué en partie la parole d'un certain groupe d'interlocuteurs, là où l'aide apportée à la remplaçante a facilité les échanges entre nous pour les jours à venir. Les dons symboliques en fonction des différentes postures ne sont pas sans incidence sur le déroulement du travail de terrain.

\section{Positionnement sur le terrain : questions préalables, questions induites?}

Il a donc fallu s'adapter, négocier sans cesse avec les personnes du terrain, inventer les conduites à adopter avec les autres, mais aussi avec moi-même. Alors que l'on tente de saisir des représentations d'ordre sociolinguistique dans le cadre de sa recherche, on est en train, au même moment, d'appréhender des représentations éventuelles, des lieux communs et stéréotypes de soi-même, ce qui peut sembler pour le moins paradoxal.

Les questions de rapport au terrain sont traitées depuis longtemps en sciences humaines et sociales (surtout en ethnologie et en sociologie), et font toujours l'objet de réactualisations par l'exercice de l'auto-analyse, encore appelée socio-analyse, dont l'idée forte est la réflexivité, c'est à dire un regard critique sur soi en action. Voici ce qu'en dit Florence Weber dans un entretien: "Il m'a semblé indispensable de comprendre la façon dont j'avais observé pour pouvoir tirer les leçons de mes observations elles-mêmes. » ; " Quand on se regarde en train de travailler, est-ce qu'on travaille encore de la même façon? » (Noiriel \& Weber, 1990 [12]).

Ceci rejoint un paradoxe de l'observation participante dans le sens où lorsqu'on participe soi-même aux situations qui produisent des données, l'analyse de sa posture est également à analyser (Labov, 1976 [13]).Et pour rejoindre mon propos aujourd'hui, si les postures sont multiples, l'analyse de cette multiplicité est nécessaire, tant dans la construction que dans l'analyse des données. Nous sommes donc au cœur du cadre conceptuel global. Qui se construit sous forme de «trajets » permanents entre revue de littérature, postures épistémologiques et découvertes empiriques.

Aux questions: Comment conserve-t-on son intégrité lorsqu'on essaye de s'adapter? Est-il scientifiquement possible de se saisir de ses compétences plurielles pour sa recherche ? Humainement, peut-on être des deux côtés de la barrière symbolique souvent existante entre enseignants et apprenants ? Certains éléments de réponse apparaissent à travers la synergie entre l'élaboration de l'objet de recherche, la mise en place du cadre théorique, le déroulement du terrain et les premières analyses. L'enquête n'a donc pas été menée de façon linéaire et de nombreux allers-retours et ont été effectués afin de réajuster et d'affiner les relations entre les acteurs ainsi que la problématique même de la thèse.

Les négociations se construisent et dépendent des facultés d'adaptation des locuteurs, notamment de ceux qui sont engagés dans l'interaction. Ainsi, l'approche des représentations sociolinguistiques est passée par une posture compréhensive et un exercice (dans le sens d'expérience et de métier d'ethnosociolinguiste) de saisie des négociations interactives et des échanges symboliques. Pour reprendre les 
propos de Goffman : «l'acteur doit agir de façon à donner, intentionnellement ou non, une expression de lui-même, et les autres, à leur tour doivent en retirer une certaine impression » (Goffman, 1973 [2]).

Mais plus qu'une question de réflexivité, dans laquelle il me semble que la part faite aux rapports aux autres n'est pas assez explicite (il serait possible de s'auto-analyser tout au long de ses recherches, mais pour quoi, pour produire quoi ?), il me semble que ce qui reflète le plus justement la complexité des relations sur le terrain est le terme d'altéro-réflexivité que Didier de Robillard définit comme étant : « une dialectique de la nécessaire projection du chercheur dans son travail d'élucidation du sens pour lui, pour son projet de recherche, et de la prise en compte de ses destinataires potentiels. L'altéroréflexivité suscite une instabilisation de perspectives, qui fait faire au chercheur une expérience d'altérité lui ouvrant des perspectives, d'autres points de vue à partir desquels il peut construire des postures nouvelles » (de Robillard, 2007[14]).

\section{Conclusion}

Mener une recherche dans laquelle le travail de terrain, le rapport à l'Autre est prépondérant signifie apprendre à appliquer et s'appliquer une éthique scientifique mais aussi une éthique des relations interpersonnelles (entre les participants et le chercheur) (Blanchet, 2000 [1], Rabatel, 2013 [15]). Cette éthique globale n'étant pas une recette utilisable clé en main, mais bel et bien un travail personnel et scientifique se construisant sur des allers-retours permanents entre revue de littérature et rencontre avec les acteurs du champ préalablement défini, il semble nécessaire d'expliciter ses choix et ses postures («je parle d'où? ») afin de pouvoir poser une analyse pertinente mais aussi située.

L'exercice de (re)présentation du jeune chercheur se présenterait alors sous la forme d'une coconstruction, entre négociations et réflexions épistémologiques, avec la conscience qu'il n'est pas interchangeable, avec ce que cela implique de confiance en soi, de prise de risque et de légitimité.

\section{Bibliographie}

1. P. Blanchet, Linguistique de terrain : méthode et théorie : une approche ethno-sociolinguistique (2000).

2. E. Goffman, La mise en scène de la vie quotidienne, tome 1 : La présentation de soi (1973).

3. H.S. Becker, Les ficelles du métier : comment conduire sa recherche en sciences sociales (2002).

4. P. Paillé, A. Mucchielli, L'analyse qualitative en sciences humaines et sociales (2012).

5. J. Meyer, Accents et discriminations : entre variation linguistique et marqueurs identitaires. Cahiers internationaux de sociolinguistique, 1, 33-51. (2012).

6. M. Gasquet-Cyrus, «L'accent : concept (socio)linguistique ou catégorie de sens commun? » in H. Boyer (Dir.) Pour une épistémologie de la sociolinguistique p. 179-189 (2010).

7. D. Moore (coord.), Les représentations des langues et de leur apprentissage (2001).

8. H. Adami, S. Etienne, A. Bretegnier (Dir.), Littéracie et alphabétisation des adultes : questions théoriques, andragogiques et didactiques. Savoirs et Formation, Recherches et Pratiques, 1 (2010).

9. A. Madec, Rires et relations d'enquête, Ethnologie française 32, 89-94 (2002).

10. A. Bretegnier, «Histoires de langues en formation, Une démarche de recherche-intervention formative alter-réflexive» in M. Molinié \& E. Huver (coord.), Praticiens - chercheurs à l'écoute du sujet plurilingue. Réflexivité et interaction biographique en sociolinguistique et en didactique, CAS 4 (2009).

11. A. Bretegnier, « Rapports aux langues, relations aux langues : une histoire interactionnelle », in M. Dreyfus et J.-M. Prieur (Dirs.), Hétérogénéité et variation. Perspectives sociolinguistiques, didactiques et anthropologiques, 196-206 (2012). 
12. G. Noiriel, Journal de terrain, journal de recherche et auto-analyse. Entretien avec Florence Weber. Genèses, 2. A la découverte du fait social, 138-147 (1990).

13. W. Labov, Sociolinguistique, 1976.

14. D. (de) Robillard, La linguistique autrement : altérité, expérienciation, réflexivité, constructivisme, multiversalité : en attendant que le Titanic ne coule pas in Ph. Blanchet/ L.-J. Calvet / D. de Robillard, Un siècle après le Cours de Saussure : la linguistique en question, CAS, 1, 81-228 (2007).

15. A. Rabatel, L'engagement $d u$ chercheur, entre "éthique d'objectivité " et "éthique de subjectivité », Argumentation et Analyse du Discours (2013). 